\title{
Flux tubes in the QCD vacuum
}

\author{
Paolo Cea* \\ Dipartimento di Fisica dell'Università di Bari, I-70126 Bari, Italy \\ and INFN - Sezione di Bari, I-70126 Bari, Italy \\ Leonardo Cosmai ${ }^{\dagger}$ \\ INFN - Sezione di Bari, I-70126 Bari, Italy \\ Francesca Cuteri $i^{\ddagger}$ \\ Institut für Theoretische Physik, Goethe Universität, \\ 60438 Frankfurt am Main, Germany \\ Alessandro $\mathrm{Papa}^{\S}$ \\ Dipartimento di Fisica dell'Università della Calabria, \\ I-87036 Arcavacata di Rende, Cosenza, Italy \\ and INFN - Gruppo collegato di Cosenza, \\ I-87036 Arcavacata di Rende, Cosenza, Italy
}

(Dated: August 28, 2018)

\begin{abstract}
The hypothesis that the QCD vacuum can be modeled as a dual superconductor is a powerful tool to describe the distribution of the color field generated by a quark-antiquark static pair and, as such, can provide useful clues for the understanding of confinement. In this work we investigate, by lattice Monte Carlo simulations of the $S U(3)$ pure gauge theory and of $(2+1)$-flavor QCD with physical mass settings, some properties of the chromoelectric flux tube at zero temperature and their dependence on the physical distance between the static sources. We draw some conclusions about the validity domain of the dual superconductor picture.
\end{abstract}

PACS numbers: 11.15.Ha, 12.38.Aw

\footnotetext{
* paolo.cea@ba.infn.it

$\dagger$ leonardo.cosmai@ba.infn.it

$\ddagger$ lcuteri@th.physik.uni-frankfurt.de

$\S$ papa@cs.infn.it
} 


\section{INTRODUCTION}

The confinement of quarks and gluons inside hadrons is a well established experimental fact, but a theoretical explanation of the underlying dynamics within the theory of strong interactions, Quantum ChromoDynamics (QCD), is still missing. Numerical simulations by Monte Carlo methods of QCD on a space-time lattice provide us with a powerful nonperturbative tool to probe the vacuum structure of the theory and can help us in catching some relevant information at the basis of the confinement phenomenon.

One well established fact, ascertained by a wealth of numerical analyses in QCD, is that the chromoelectric field between two static quarks distributes in tubelike structures or "flux tubes" [1-24]. From these tubelike structures a linear potential between static color charges naturally arises, thus representing a numerical evidence of color confinement [25, 26].

As for a possible dynamical mechanism for confinement, long ago 't Hooft [27] and Mandelstam [28] conjectured that the vacuum of QCD could behave as a coherent state of color magnetic monopoles or, in more modern terms, as a dual superconductor [29, 30]: the condensation of color magnetic monopoles would play in the QCD vacuum the same role as the condensation of Cooper pairs in a standard superconductor. Indeed, there is a lot of numerical evidence in favor of color magnetic condensation [31-40], however it cannot be excluded that color magnetic monopole condensation is a consequence of the mechanism of color confinement [41], whose origin could be found in some, so far unknown, dynamical effect. Still, the dual superconductivity picture of the QCD vacuum can serve as a very useful phenomenological tool to interpret the vacuum dynamics. Many previous studies of our collaboration (or of a part of it) [10-14, 18-22] have indeed furnished clear evidence that, at zero temperature, color flux tubes, made up almost completely by the longitudinal chromoelectric field directed along the line joining a static quark-antiquark pair, can be successfully described within the dual superconductivity picture, both in $S U(2)$ and in $S U(3)$ pure gauge theories. In our most recent paper [42] the investigation of the structure of flux tubes in $S U(3)$ was extended to the case of nonzero temperature and lead to the result that the flux tube between two static sources separated by a distance of about $0.76 \mathrm{fm}$ survives even above the critical temperature $T_{c}$ of the deconfinement transition, keeping a more or

less constant transverse shape, but housing in it a weaker and weaker chromoelectric field 
as the temperature increases. Such (somewhat surprising) phenomenon could be peculiar of the only value of the distance between the sources considered in that work and evidently motivates to extend the analysis to different values of the distance between the sources. As a matter of fact, a careful study, within the dual superconductor model, of the dependence of the flux tube shape on the distance between the color sources has not been carried out so far either at zero temperature, at least in non-Abelian lattice field theories. In the threedimensional Abelian U(1) lattice gauge theory, instead, such an analysis has been completed very recently [43].

The role of the distance $d$ between the static sources for the distribution of the color fields and, hence, for the shape of the flux tube has been emphasized in [44]: at small distances the dual superconductivity picture is expected to hold, whereas the effective string theory approach [45-47] is expected to take over at large distances, the transition regime being localized around $d=2 / \sqrt{\sigma}$. According to the effective string theory description, the shape of the flux tube is determined by a fluctuating thin string connecting the sources. Within this approach the quark-antiquark potential and the width of the flux tube have been studied numerically in $S U(N)$ gauge theories, both at $T=0$ and at $T<T_{c}$, in many papers [48-54]. In several other recent works [23,55-60] also the detailed profile of the color field distribution near static sources has been analyzed.

The aim of this paper is to assess the validity domain of the dual superconductivity picture of the QCD vacuum, by confronting its predictions for some of the parameters determining the shape of the flux tube with Monte Carlo data, when the distance $d$ between the static sources is varied in the range $0.76 \mathrm{fm}$ to $1.33 \mathrm{fm}$, corresponding to the range $1.6 / \sqrt{\sigma}$ to $2.8 / \sqrt{\sigma}$. The analysis is performed both in the $S U(3)$ pure gauge theory and, for the first time ever, also in (2+1)-flavor QCD with physical quark mass settings. The considered range of distances is the largest one for which the setup of our numerical analysis allowed the extraction of physical information out of the statistical noise and is large enough to include the regime where, according to [44], the dual superconductivity hypothesis should fail.

The plan of the paper is the following: in Section II we recall the theoretical background for the dual superconductivity predictions and introduce the lattice observables used to extract the field strength tensor of the static quark-antiquark sources; in Section III we illustrate our lattice setup and present the numerical results of our analysis; finally, in 
Section IV, we comment on our findings.

\section{THEORETICAL BACKGROUND AND LATTICE OBSERVABLES}

The field configurations generated by a static quark-antiquark pair can be probed by calculating on the lattice the vacuum expectation value of the following connected correlation function $[5,6,61,62]$ :

$$
\rho_{W, \mu \nu}^{\mathrm{conn}}=\frac{\left\langle\operatorname{tr}\left(W L U_{P} L^{\dagger}\right)\right\rangle}{\langle\operatorname{tr}(W)\rangle}-\frac{1}{N} \frac{\left\langle\operatorname{tr}\left(U_{P}\right) \operatorname{tr}(W)\right\rangle}{\langle\operatorname{tr}(W)\rangle} .
$$

Here $U_{P}=U_{\mu \nu}(x)$ is the plaquette in the $(\mu, \nu)$ plane, connected to the Wilson loop $W$, lying on the $\hat{4} \hat{i}$-plane, with $\hat{i}$ any fixed spatial direction, by a Schwinger line $L$, and $N$ is the number of colors. The correlation function defined in Eq. (1) measures the field strength,

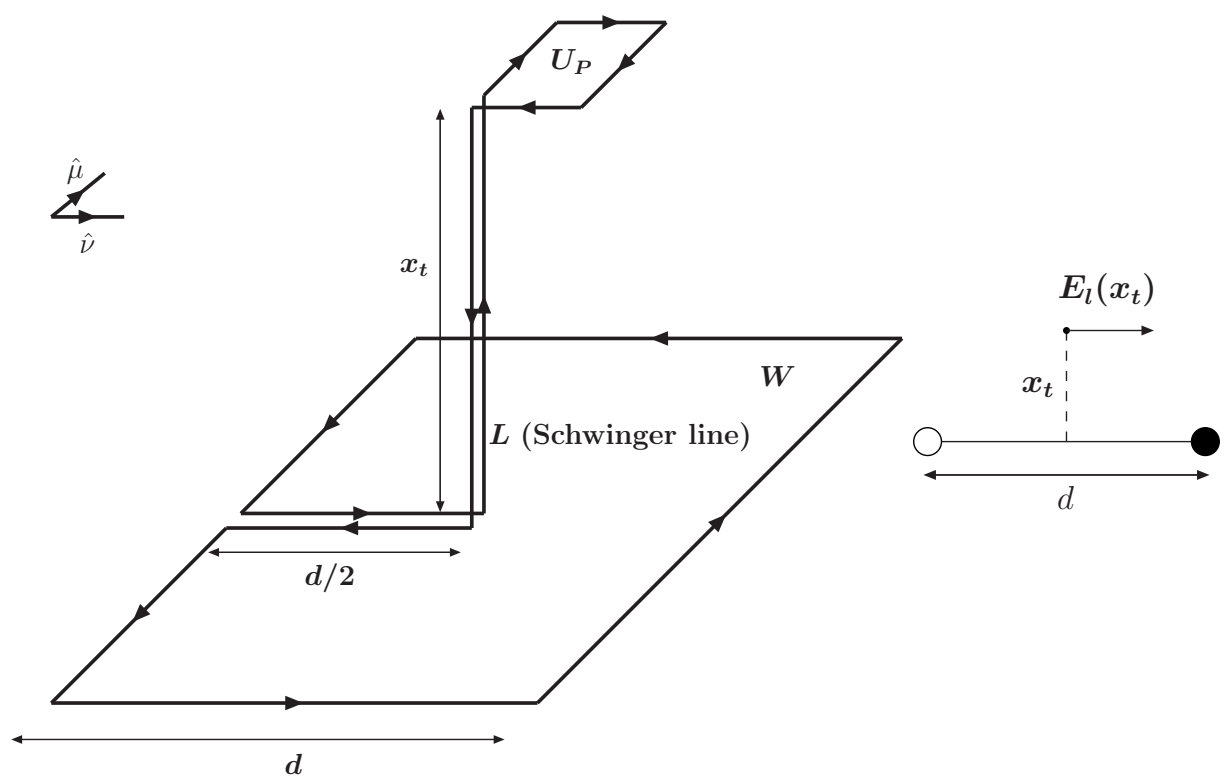

FIG. 1: (Left) The connected correlator given in Eq. (1) between the plaquette $U_{P}$ and the

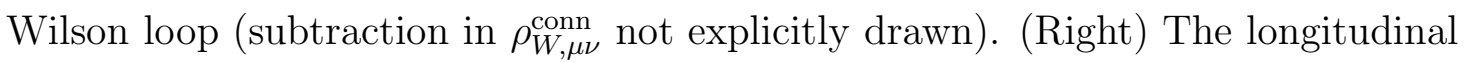
chromoelectric field $E_{l}\left(x_{t}\right)$ with respect to the position of the static sources (represented by the white and black circles), for a given value of the transverse distance $x_{t}$.

since in the naive continuum limit [6]

$$
\rho_{W, \mu \nu}^{\operatorname{conn}} \stackrel{a \rightarrow 0}{\longrightarrow} a^{2} g\left[\left\langle F_{\mu \nu}\right\rangle_{q \bar{q}}-\left\langle F_{\mu \nu}\right\rangle_{0}\right]
$$


where \langle\rangle$_{q \bar{q}}$ denotes the average in the presence of a static $q \bar{q}$ pair and \langle\rangle$_{0}$ is the vacuum average, which is expected to vanish. This leads to the following definition of the quarkantiquark field strength tensor:

$$
F_{\mu \nu}(x)=\frac{1}{a^{2} g} \rho_{W, \mu \nu}^{\mathrm{conn}}(x)
$$

In the particular case when the plaquette $U_{P}$ and the Wilson loop $W$ lie on parallel planes (see Fig. 1 (left) with $\hat{\mu}=\hat{4}$ and $\hat{\nu}=\hat{i}$ ), we get $F_{4 i}(x)$, i.e. the chromoelectric field in the direction $\hat{i}$, which is the direction longitudinal to the axis connecting the two static sources. The position $x$ in space where the longitudinal chromoelectric field is evaluated depends on the relative position of the plaquette $U_{P}$ to the Wilson loop $W$; for the setup of Fig. 1 (left), the point $x$ is equidistant to the two static sources and off the axis connecting them (no matter in which direction, due to the azimuthal symmetry) by a distance $x_{t}$. In the following we denote the longitudinal chromoelectric field $F_{4 i}(x)$ by $E_{l}\left(x_{t}\right)$ (see also Fig. 1 (right)).

In this study we have not considered the effect of changing the path along which the Schwinger line $L$ is constructed. However, in a study about gauge-invariant field-strength correlators [63] the dependence on the shape of the Schwinger line was investigated and it was found that, while different shapes correspond to differences in the intensity of the measured field, the slope seemed to be completely path independent. The same path independence is then plausible for all the physical quantities extracted from our fits.

As far as the color structure of the field $F_{\mu \nu}$ is concerned, we observe that the Wilson loop connected to the plaquette is the source of a color field which points, in average, onto an unknown direction $n^{a}$ in color space (there is no preferred direction). We thus measure the average projection of the color field onto that direction. The role of the Schwinger lines entering the definition (1) is to realize the color parallel transport between the source loop and the "probe" plaquette. Therefore, the $F_{\mu \nu}$ appearing in Eq. (3), should be understood as $n^{a} F_{\mu \nu}^{a}$,

$$
\rho_{W, \mu \nu}^{\operatorname{conn}} \stackrel{a \rightarrow 0}{\longrightarrow} a^{2} g\left[\left\langle n^{a} F_{\mu \nu}^{a}\right\rangle_{q \bar{q}}\right]
$$

This relation is a necessary consequence of the gauge-invariance of the operator defined in (1) and of its linear dependence on the color field in the continuum limit (see Eq. (2)). An explicit verification of the latter property was exhibited in Ref. [42] (see Fig. 3 there). 
The numerical results presented in this work refer to the longitudinal chromoelectric field $E_{l}\left(x_{t}\right)$ for different values of $x_{t}$ and for several choices of the distance $d$ between the static sources. Due to the azimuthal symmetry, the transverse shape of the longitudinal chromoelectric field at midway between the static sources can be fully reconstructed. It is evidently useful to describe this transverse shape in terms of a few physical parameters, which could possibly help identifying the underlying mechanism of confinement. The dual superconductor model turns out to be a powerful tool to describe this transverse shape, at least at distances $d$ not too large with respect to the inverse square root of the string tension.

The key assumption of the dual superconductor model is to understand the chromoelectric flux tube in the QCD vacuum as the dual counterpart of an Abrikosov tube inside an ordinary superconductor. According to this interpretation, the transverse shape of the longitudinal chromoelectric field $E_{l}$ should resemble the dual version of the Abrikosov vortex field distribution. This naturally leads to the idea of describing chromoelectric flux tubes by means of the same tube-like solutions of the Ginzburg-Landau equations in usual electric superconductivity. One such solution was proposed long ago [8, 10-14] to fit the transverse shape of the longitudinal chromoelectric field:

$$
E_{l}\left(x_{t}\right)=\frac{\phi}{2 \pi} \mu^{2} K_{0}\left(\mu x_{t}\right), \quad x_{t}>0
$$

Here $K_{n}$ is the modified Bessel function of order $n, \phi$ is the external flux, and $\lambda=1 / \mu$ is the London penetration length. This field shape in Eq. (5) is acceptable provided that $\lambda \gg \xi$, $\xi$ being the coherence length which represents the typical size scale of the density variations of the magnetic monopole condensate (the dual version of the Cooper condensate). Due to this condition, the solution given in (5) is appropriate only for superconductors that, in the language of ordinary superconductivity, are classified as type-II superconductors. However, within the dual superconductor model it is expected that, having the source and sink of the color fields in the QCD vacuum, tube-like structures arise irrespective of the value of the $\lambda / \xi$ ratio. The main flaw of the ansatz in Eq. (5) is, instead, the divergence of the field value at $x_{t}=0$. In this respect a more adequate solution was constructed long ago in Ref. [64], where, starting from a simple variational model for the magnitude of the normalized order parameter of an isolated vortex, an analytic expression was derived for both the magnetic field and supercurrent density, that solves Ampère's law and the Ginzburg-Landau equations. Only recently it was suggested and successfully adopted [19-22] in order to describe the transverse 
distribution of the chromoelectric flux tube:

$$
E_{l}\left(x_{t}\right)=\frac{\phi}{2 \pi} \frac{1}{\lambda \xi_{v}} \frac{K_{0}(R / \lambda)}{K_{1}\left(\xi_{v} / \lambda\right)}
$$

where

$$
R=\sqrt{x_{t}^{2}+\xi_{v}^{2}}
$$

and $\xi_{v}$ is a variational core-radius parameter. Equation (6) can be rewritten as

$$
E_{l}\left(x_{t}\right)=\frac{\phi}{2 \pi} \frac{\mu^{2}}{\alpha} \frac{K_{0}\left[\left(\mu^{2} x_{t}^{2}+\alpha^{2}\right)^{1 / 2}\right]}{K_{1}[\alpha]}
$$

with

$$
\mu=\frac{1}{\lambda}, \quad \frac{1}{\alpha}=\frac{\lambda}{\xi_{v}} .
$$

By fitting Eq. (8) to flux-tube data, one can get both the penetration length $\lambda$ and the ratio of the penetration length to the variational core-radius parameter, $\lambda / \xi_{v}$. Moreover, the Ginzburg-Landau $\kappa$ parameter, which in ordinary superconductivity discriminates the type of superconductor, can be obtained by

$$
\kappa=\frac{\lambda}{\xi}=\frac{\sqrt{2}}{\alpha}\left[1-K_{0}^{2}(\alpha) / K_{1}^{2}(\alpha)\right]^{1 / 2},
$$

whereas the coherence length $\xi$ can be determined by combining Eqs. (9) and (10). We will consider two more observables which give information about the structure and the properties of the chromoelectric flux tube: the mean square root width,

$$
\sqrt{w^{2}}=\sqrt{\frac{\int d^{2} x_{t} x_{t}^{2} E_{l}\left(x_{t}\right)}{\int d^{2} x_{t} E_{l}\left(x_{t}\right)}}=\sqrt{\frac{2 \alpha}{\mu^{2}} \frac{K_{2}(\alpha)}{K_{1}(\alpha)}}
$$

and the square root of the energy per unit length, normalized to the flux $\phi$,

$$
\frac{\sqrt{\varepsilon}}{\phi}=\frac{1}{\phi} \sqrt{\int d^{2} x_{t} \frac{E_{l}^{2}\left(x_{t}\right)}{2}}=\sqrt{\frac{\mu^{2}}{8 \pi}\left(1-\left(\frac{K_{0}(\alpha)}{K_{1}(\alpha)}\right)^{2}\right)} .
$$

\section{LATTICE SETUP AND NUMERICAL RESULTS}

We performed all simulations, both for pure gauge $S U(3)$ and $(2+1)$-flavor QCD, on $32^{4}$ lattices, making use of the publicly available MILC code [65], suitably modified in order to introduce the relevant observables. The typical statistics of each run was about 4000-5000; 
to allow for thermalization we typically discarded a few thousand sweeps. The error analysis was performed by the jackknife method over bins at different blocking levels.

The lattice discretization that we used for the pure gauge $S U(3)$ is the standard Wilson action, with the physical scale set assuming for the string tension the standard value of $\sqrt{\sigma}=420 \mathrm{MeV}$ and using the parameterization [66]

$$
\begin{aligned}
& (a \sqrt{\sigma})(g)=f_{\mathrm{SU}(3)}\left(g^{2}\right)\left\{1+0.2731 \hat{a}^{2}(g)\right. \\
& \left.-0.01545 \hat{a}^{4}(g)+0.01975 \hat{a}^{6}(g)\right\} / 0.01364, \\
& \hat{a}(g)=\frac{f_{\mathrm{SU}(3)}\left(g^{2}\right)}{f_{\mathrm{SU}(3)}\left(g^{2}(\beta=6)\right)}, \beta=\frac{6}{g^{2}}, \quad 5.6 \leq \beta \leq 6.5,
\end{aligned}
$$

with

$$
f_{\mathrm{SU}(3)}\left(g^{2}\right)=\left(b_{0} g^{2}\right)^{-b_{1} / 2 b_{0}^{2}} \exp \left(-\frac{1}{2 b_{0} g^{2}}\right), \quad b_{0}=\frac{11}{(4 \pi)^{2}}, \quad b_{1}=\frac{102}{(4 \pi)^{4}} .
$$

The used value of the string tension comes from the universal slope of the Regge trajectories and the phenomenology of heavy quark systems [67]. For (2+1)-flavor QCD we adopted the HISQ/tree action [68] and worked on the line of constant physics determined in [69], by adjusting the coupling and the bare quark masses so as to keep the strange quark mass $m_{s}$ fixed at its physical value with the light-to-strange mass ratio $m_{l} / m_{s}=1 / 20$, corresponding to a pion mass of $160 \mathrm{MeV}$. The scale was set through the slope of the static quark-antiquark potential evaluated on zero-temperature lattices, using the results of Ref. [69].

\section{A. Smoothing procedure}

The connected correlator defined in Eq. (1) suffers from large fluctuations at the scale of the lattice spacing, which are responsible for a bad signal-to-noise ratio. To extract the physical information carried by fluctuations at the physical scale (and, therefore, at large distances in lattice units) we smoothed out configurations by the smearing procedure. Our setup consisted of (just) one step of HYP smearing [70] on the temporal links, with smearing parameters $\left(\alpha_{1}, \alpha_{2}, \alpha_{3}\right)=(1.0,0.5,0.5)$, and $N_{\mathrm{APE}}$ steps of APE smearing [71] on the spatial links, with smearing parameter $\alpha_{\mathrm{APE}}=0.167$. Here $\alpha_{\mathrm{APE}}$ is the ratio between the weight of one staple and the weight of the original link. The optimal number of smearing step was found by looking at the smearing step at which our direct observable $E_{l}\left(x_{t}\right)$ showed the largest signal-to-noise ratio, with the smearing parameter tuned in such a way that in the $E_{l}\left(x_{t}\right)$ vs 'smearing step' plot we could see a clear plateau. 
In Fig. 2 we show the behavior under smearing of the longitudinal chromoelectric field $E_{l}\left(x_{t}\right)$ in pure gauge $S U(3)$ for a physical distance between the static sources equal to $0.76 \mathrm{fm}$. We can see that, for each value of the distance $x_{t}$ in the direction transverse to the axis connecting the sources, a clear plateau is reached after a sufficiently large number of smearing steps. A similar behavior was observed in all simulations we performed, both in pure gauge $S U(3)$ and in (2+1)-flavor QCD. All results concerning the chromoelectric field $E_{l}\left(x_{t}\right)$ presented in the following will always refer to determinations on smeared configurations, after a number of smearing steps $N_{\mathrm{APE}}$ such that the plateau is reached for all considered values of $x_{t}$. The typical value of $N_{\mathrm{APE}}$ ranges between 25 and 50 .

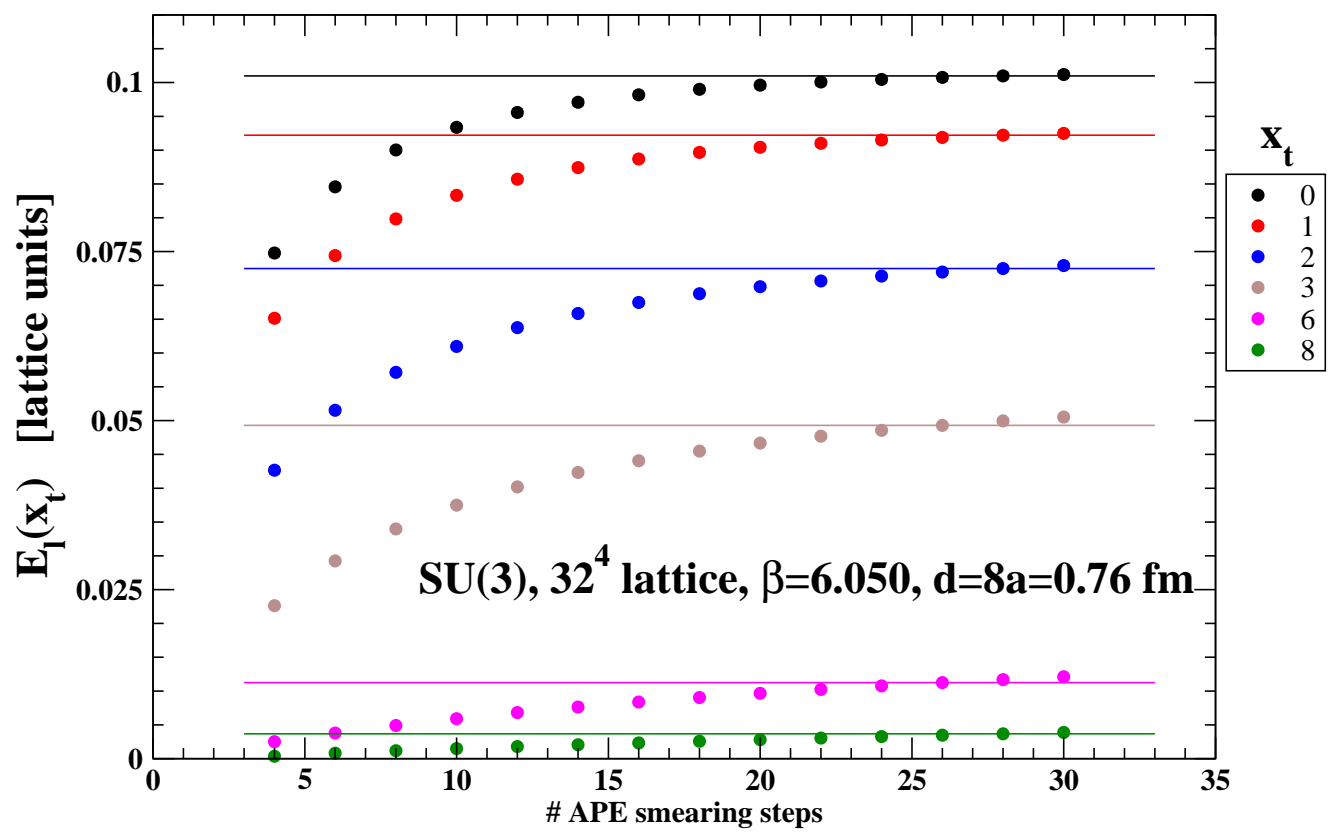

FIG. 2: (color online). Behavior of the longitudinal chromoelectric field $E_{l}$, on a given lattice and for various values of the distance from the axis connecting the static sources, versus the number of APE smearing steps on the spatial links.

\section{B. Continuum scaling}

Our aim is to determine the physical properties of the chromoelectric flux tube in the continuum, for this reason, we have preliminarily checked that our simulations are performed in a region of values of the coupling $\beta$ where continuum scaling holds.

We have hence measured the longitudinal chromoelectric field generated when the static 
sources are located at the same physical distance $d$, but for two different values of the coupling $\beta$ or, equivalently, of the distance in lattice units. This test was performed both in pure gauge $S U(3)$ and in $(2+1)$-flavor QCD.

In Fig. 3 we present the outcome of this test: in the left panel we show the (smeared) chromoelectric field in pure gauge $S U(3)$ versus the transverse distance $x_{t}$ in physical units, when the sources are placed at distance $8 a$ and $10 a$ ( $a$ is the lattice spacing) at $\beta=6.050$ and $\beta=6.195$, respectively, so that, according to Eq. (13), the physical distance is, in both cases, equal to $0.76 \mathrm{fm}$ in physical units. The right panel of Fig. 3 shows the result of a similar analysis in (2+1)-flavor QCD: here the distances in lattice units were fixed at distances $7 a$ and $8 a$, for $\beta=6.743$ and $\beta=6.885$, respectively, so that the physical distance $d$ between the sources is again equal to $0.76 \mathrm{fm}$. In both cases an almost perfect scaling can be observed, thus making us confident that, for the observable of interest in this work, the continuum scaling is reached in $S U(3)$ (at least) for $\beta=6.050$ and in (2+1)-flavor QCD (at least) for $\beta=6.743$. Another hint from the results shown in Fig. 3 is that our smearing procedure is robust: had the smearing procedure badly corrupted the physical signal for the chromoelectric field, it would have been quite unlikely to obtain such a nice scaling.
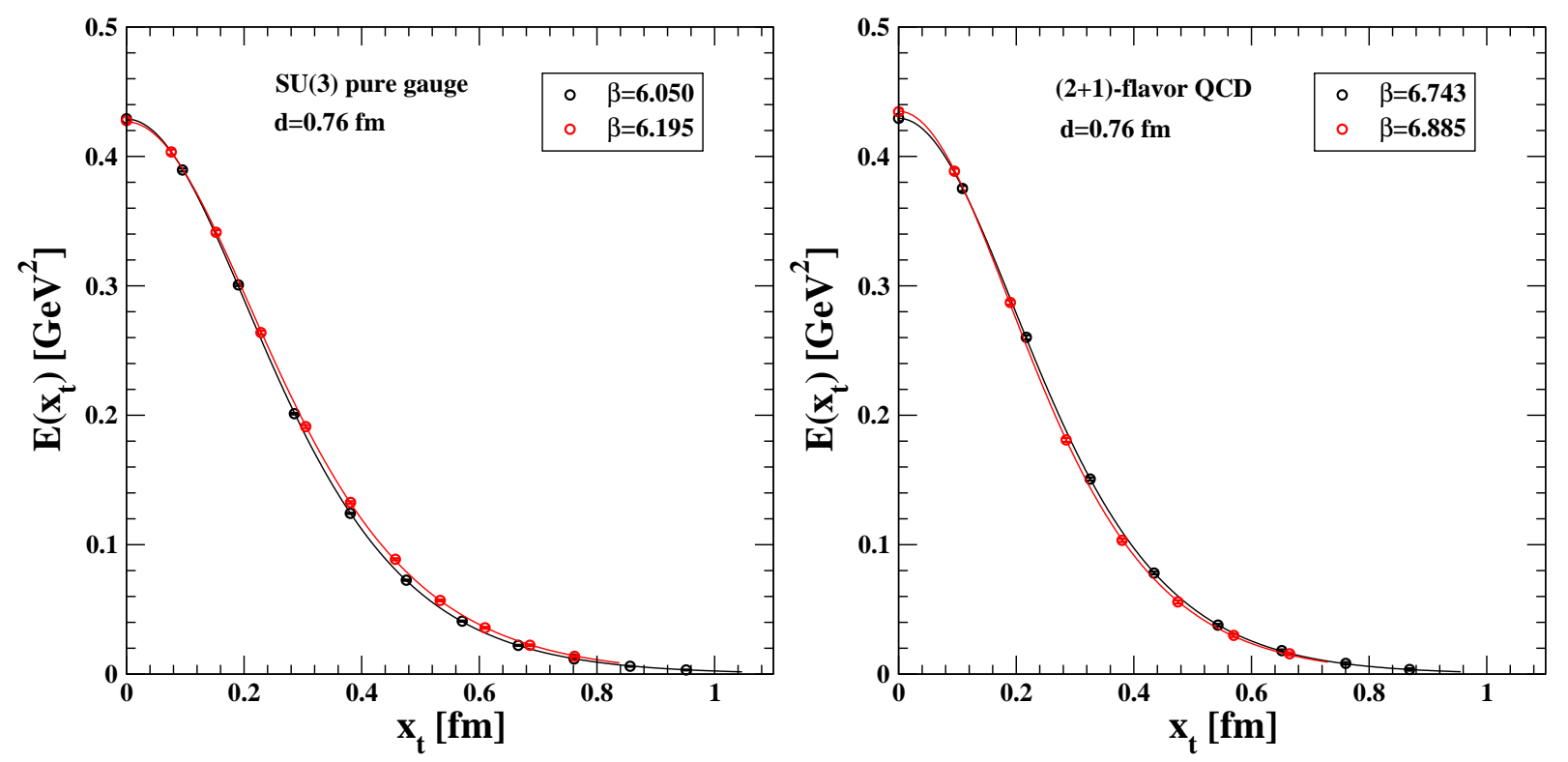

FIG. 3: (color online). Behavior of the longitudinal chromoelectric field $E_{l}$ (in physical units) versus the distance $x_{t}$ (in physical units) from the axis connecting the static sources, at the same physical distance between the sources as obtained for two different $\beta$ values, in the case of the $S U(3)$ pure gauge theory (left panel) and of (2+1)-flavor QCD (right panel). 

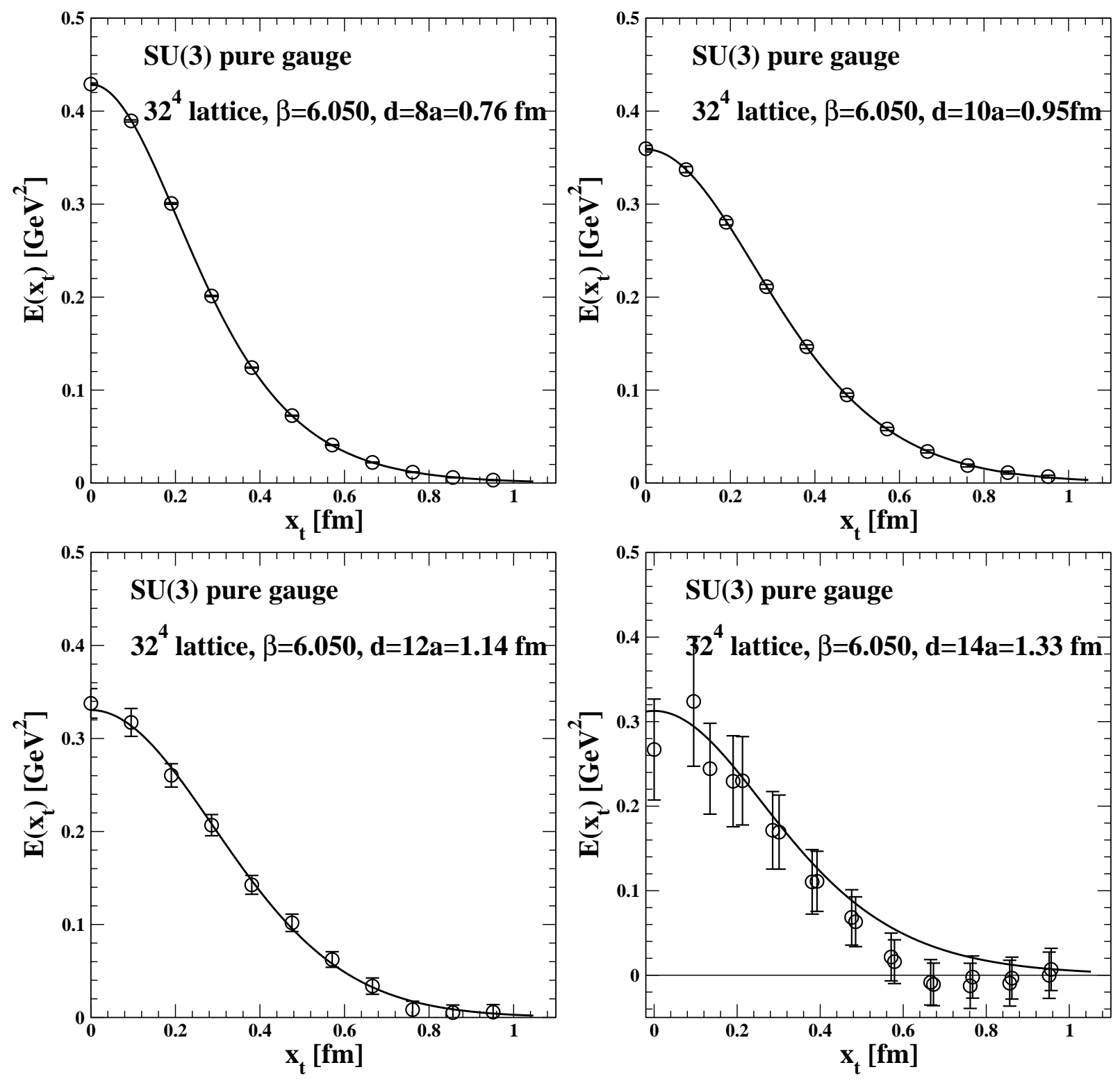

FIG. 4: Behavior of the longitudinal chromoelectric field $E_{l}$ (in physical units) versus the distance $x_{t}$ (in physical units) from the axis connecting the static sources, at four different values of the physical distance between the sources in the $S U(3)$ pure gauge theory.

\section{Shape of the flux tube}

We determined, both in $S U(3)$ and in $(2+1)$-flavor QCD, the dependence of the longitudinal chromoelectric field $E_{l}$ on the transverse distance $x_{t}$, by Monte Carlo evaluations of the expectation value of the operator $\rho_{W, \mu \nu}^{\text {conn }}$ (see Eq. (1)) over smeared ensembles, and compared it with the function given in Eq. (8). Such comparison was carried out for a few 
TABLE I: Flux tube parameters for the $S U(3)$ pure gauge theory at various distances between the static sources.

\begin{tabular}{|c|c|c|c|c|c|c|c|}
\hline \hline$\beta$ & $d[\mathrm{fm}]$ & $\phi$ & $\lambda[\mathrm{fm}]$ & $\kappa=\lambda / \xi$ & $\xi[\mathrm{fm}]$ & $\sqrt{w^{2}}[\mathrm{fm}]$ & $\sqrt{\varepsilon} / \phi[\mathrm{GeV}]$ \\
\hline 6.050 & 0.76 & $5.143(39)$ & $0.164(5)$ & $0.348(208)$ & $0.472(283)$ & $0.458(17)$ & $0.133(5)$ \\
6.195 & 0.76 & $4.862(40)$ & $0.155(6)$ & $0.306(167)$ & $0.506(278)$ & $0.443(19)$ & $0.137(6)$ \\
6.050 & 0.95 & $5.287(109)$ & $0.146(17)$ & $0.170(63)$ & $0.859(331)$ & $0.479(69)$ & $0.123(19)$ \\
6.050 & 1.14 & $5.218(371)$ & $0.143(40)$ & $0.145(48)$ & $0.983(428)$ & $0.488(140)$ & $0.120(35)$ \\
6.050 & 1.33 & $5.000(292)$ & $0.169(16)$ & $0.236(109)$ & $0.715(335)$ & $0.512(114)$ & $0.117(30)$ \\
\hline
\end{tabular}

values of the distance $d$ between the static sources, at values of the $\beta$-coupling lying inside the continuum scaling region.

In Fig. 4 we report the results in physical units of our simulations for the case of the $S U(3)$ pure gauge theory: we can see that, in an interval of distances between the sources ranging from $0.76 \mathrm{fm}$ to $1.33 \mathrm{fm}$, data for $E_{l}\left(x_{t}\right)$ are nicely fitted by the function given in Eq. (8), with $\chi^{2} /$ dof $=\mathcal{O}(1)$. For larger values of $d$ the statistical noise becomes overwhelming, preventing us from extracting reliable estimates using the present setup.

The fundamental fit parameters $\lambda$ and $\xi$ are summarized in Table I, together with $\kappa=$ $\lambda / \xi$, the mean square root width, $\sqrt{w^{2}}$, and the square root of the normalized energy per unit length, $\frac{\sqrt{\varepsilon}}{\phi}$. We can argue that the penetration length $\lambda$ is stable within errors under variations of the distance between the sources, while there seems to be a slow increase of $\xi$ and $\sqrt{w^{2}}$ as $d$ grows. We notice also that the values of $\lambda$ obtained here for the case of the $S U(3)$ pure gauge theory nicely compare with our previous determination [20], obtained for a distance $d=0.62 \mathrm{fm}$ on a smaller lattice and using a slightly different setup for the smearing procedure. We find, on the other hand, that our values for $\sqrt{w^{2}}$ are a bit larger than those found for an analogous observable in the very recent analysis of Ref. [72], where the flux tube profile was determined through the disconnected plaquette-Wilson loop correlator and an ansatz different from ours was used to interpolate it. The relatively large error bars in our determinations for $\sqrt{w^{2}}$ do not allow us to make any firm statement about the possible nature of the flux-tube widening.

A similar analysis was performed in $(2+1)$-flavor QCD, where the values of $d$ were taken 

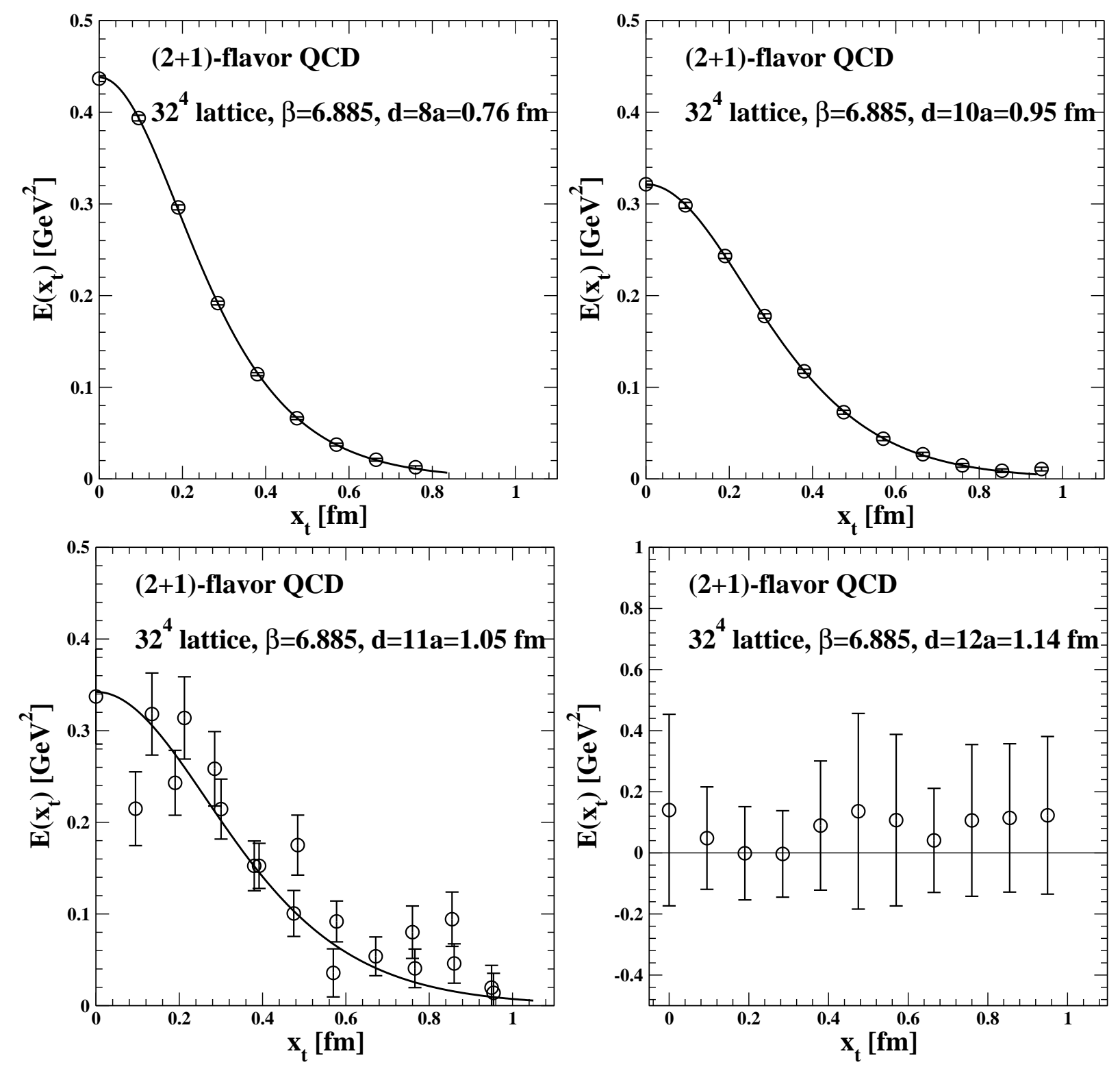

FIG. 5: Behavior of the longitudinal chromoelectric field $E_{l}$ (in physical units) versus the distance $x_{t}$ (in physical units) from the axis connecting the static sources, at four different values of the physical distance between the sources in $(2+1)$-flavor QCD.

in the range $0.76 \mathrm{fm}$ to $1.14 \mathrm{fm}$. Results are presented in Fig. 5 and in Table II. We observe first of all that at $d=0.76 \mathrm{fm}$ the value of $\lambda$ obtained in $(2+1)$-flavor QCD is fairly consistent with the one obtained in $S U(3)$. However, comparing the results at $d=0.76 \mathrm{fm}$ and at $d=0.95 \mathrm{fm}$, there is an indication that $\sqrt{w^{2}}$ keeps constant, whereas $\lambda$ increases and $\xi$ decreases. The determinations at the latter distance, however, are plagued by large uncertainties and should be handled with care. 
Another interesting fact is that at distance $d=1.14 \mathrm{fm}$, the chromoelectric longitudinal field $E_{l}\left(x_{t}\right)$ seems to fluctuate around zero, although within large error bars, while, at the same distance, a clear nonzero signal for $E_{l}\left(x_{t}\right)$ could be detected in the $S U(3)$ pure gauge theory. This circumstance could be the consequence of larger statistical fluctuations induced by dynamical fermions or be the signature of the phenomenon of "string breaking" [73, 74], which could take place just around this distance $[73,75]$.

TABLE II: Flux tube parameters for the $(2+1)$-flavor QCD at various distances between the static sources.

\begin{tabular}{|c|c|c|c|c|c|c|c|}
\hline \hline$\beta$ & $d[\mathrm{fm}]$ & $\phi$ & $\lambda[\mathrm{fm}]$ & $\kappa=\lambda / \xi$ & $\xi[\mathrm{fm}]$ & $\sqrt{w^{2}}[\mathrm{fm}]$ & $\sqrt{\varepsilon} / \phi[\mathrm{GeV}]$ \\
\hline 6.743 & 0.76 & $4.431(57)$ & $0.141(8)$ & $0.272(137)$ & $0.521(265)$ & $0.415(29)$ & $0.145(11)$ \\
6.885 & 0.76 & $4.331(82)$ & $0.155(11)$ & $0.390(252)$ & $0.398(259)$ & $0.423(34)$ & $0.145(29)$ \\
6.885 & 0.95 & $4.272(131)$ & $0.154(21)$ & $0.236(108)$ & $0.653(312)$ & $0.527(50)$ & $0.128(22)$ \\
6.885 & 1.05 & $5.580(441)$ & $0.174(14)$ & $0.236(109)$ & $0.736(343)$ & $0.527(50)$ & $0.113(11)$ \\
\hline \hline
\end{tabular}

The energy per unit length $\epsilon$, given in the last columns of Tables 1 and 2, represents the contribution of the chromoelectric field to the string tension, under the hypothesis of a uniform field along the axis of the flux tube; it turns out to be of the same order of the measured string tension, but cannot be directly compared with it, since the latter includes also the contribution from the vacuum energy and the (negligible) contribution from the other field components.

\section{DISCUSSION}

In this paper we have studied the chromoelectric field in the direction longitudinal to the line connecting a static quark-antiquark pair, and its shape on the transverse plane cutting this line in its middle point. This investigation has been performed both in the $S U(3)$ pure gauge theory and in $(2+1)$-flavor QCD, with the aim of assessing any possible effect from the variation of the physical distance between the static sources. We considered distances extending in the range $0.76 \mathrm{fm}$ to $1.33 \mathrm{fm}$.

Let us first summarize the common features we observed in the two theories: 
- the transverse shape of the longitudinal chromoelectric field midway between the sources is accessible to Monte Carlo simulations through the measurement of the expectation value of a suitable connected operator (see Eq. (1)) on smeared configuration ensembles;

- up to distances between the sources of about $1.5 \mathrm{fm}$ in the case of the $S U(3)$ pure gauge theory and of about $1.1 \mathrm{fm}$ in $(2+1)$-flavor QCD, this transverse shape is nicely described by the function (8), which is the dual version of a solution derived long ago in Ref. [64] for the magnetic field generated by a single vortex inside an ordinary superconductor;

- the values of the parameters entering this function, namely the (dual versions of) the London penetration length $\lambda$ and the coherence length $\xi$, as extracted from the fit to numerical data, indicate that the vacuum behaves as a type-I superconductor.

In the specific case of the $S U(3)$ pure gauge theory, we found that the parameter $\lambda$ and the mean width of the field transverse profile remain fairly constant under variation of the distance between the sources, whereas $\xi$ shows a tendency to increase with the distance, though within large uncertainties. The stability of $\lambda$ supports the validity of the dual superconductivity model over the considered range of distances between the sources. It would be interesting to refine the numerical techniques and to check how far sources must be located to observe the break-up of the dual superconductivity picture and the onset of the effective string description (see [44] for a nice discussion about the interplay between the two pictures).

In $(2+1)$-flavor QCD the scenario is less clean: due to the larger uncertainties and/or to the possible insurgence of new phenomena, such as the "string breaking", the range of distances we could explore is smaller than in the pure gauge theory and it is thus more difficult to identify a clear trend in the values of the parameter of the transverse field profile. Data seem to suggest that $\lambda$ increases with the distance between the sources, whereas the mean width remains stable. However, further investigations and more efficient algorithms are needed to achieve firmer conclusions. 


\section{ACKNOWLEDGMENTS}

We thank Marshall Baker for useful comments on this work. This investigation was in part based on the MILC collaboration's public lattice gauge theory code. See http://physics . utah.edu/ detar/milc.html. Numerical calculations have been made possible through a CINECA-INFN agreement, providing access to resources on GALILEO and MARCONI at CINECA.

[1] M. Fukugita and T. Niuya, Phys. Lett. B132, 374 (1983).

[2] J. E. Kiskis and K. Sparks, Phys. Rev. D30, 1326 (1984).

[3] J. W. Flower and S. W. Otto, Phys. Lett. B160, 128 (1985).

[4] J. Wosiek and R. W. Haymaker, Phys. Rev. D36, 3297 (1987).

[5] A. Di Giacomo, M. Maggiore, and S. Olejnik, Phys. Lett. B236, 199 (1990).

[6] A. Di Giacomo, M. Maggiore, and S. Olejnik, Nucl. Phys. B347, 441 (1990).

[7] V. Singh, D. A. Browne, and R. W. Haymaker, Phys. Lett. B306, 115 (1993), arXiv:heplat/9301004.

[8] P. Cea and L. Cosmai, Nucl. Phys. Proc. Suppl. 30, 572 (1993).

[9] Y. Matsubara, S. Ejiri, and T. Suzuki, Nucl. Phys. Proc. Suppl. 34, 176 (1994), arXiv:heplat/9311061.

[10] P. Cea and L. Cosmai, Nuovo Cim. A107, 541 (1994), arXiv:hep-lat/9210030.

[11] P. Cea and L. Cosmai, Nucl. Phys. Proc. Suppl. 34, 219 (1994), arXiv:hep-lat/9311023.

[12] P. Cea and L. Cosmai, Phys. Lett. B349, 343 (1995), arXiv:hep-lat/9404017.

[13] P. Cea and L. Cosmai, Nucl. Phys. Proc. Suppl. 42, 225 (1995), arXiv:hep-lat/9411048.

[14] P. Cea and L. Cosmai, Phys. Rev. D52, 5152 (1995), arXiv:hep-lat/9504008.

[15] G. S. Bali, K. Schilling, and C. Schlichter, Phys. Rev. D51, 5165 (1995), arXiv:heplat/9409005.

[16] R. W. Haymaker and T. Matsuki, Phys. Rev. D75, 014501 (2007), arXiv:hep-lat/0505019.

[17] A. D'Alessandro, M. D’Elia, and L. Tagliacozzo, Nucl.Phys. B774, 168 (2007), arXiv:heplat/0607014 [hep-lat]. 
[18] M. S. Cardaci, P. Cea, L. Cosmai, R. Falcone, and A. Papa, Phys.Rev. D83, 014502 (2011), arXiv:1011.5803 [hep-lat].

[19] P. Cea, L. Cosmai, and A. Papa, Phys.Rev. D86, 054501 (2012), arXiv:1208.1362 [hep-lat].

[20] P. Cea, L. Cosmai, F. Cuteri, and A. Papa, in Proceedings, 31st International Symposium on Lattice Field Theory (Lattice 2013), Vol. LATTICE2013 (2013) p. 468, arXiv:1310.8423 [hep-lat].

[21] P. Cea, L. Cosmai, F. Cuteri, and A. Papa, Phys. Rev. D89, 094505 (2014), arXiv:1404.1172 [hep-lat].

[22] P. Cea, L. Cosmai, F. Cuteri, and A. Papa, Proceedings, 32nd International Symposium on Lattice Field Theory (Lattice 2014), PoS LATTICE2014, 350 (2014), arXiv:1410.4394 [hep-lat].

[23] N. Cardoso, M. Cardoso, and P. Bicudo, Phys. Rev. D88, 054504 (2013), arXiv:1302.3633 [hep-lat].

[24] M. Caselle, M. Panero, R. Pellegrini, and D. Vadacchino, JHEP 01, 105 (2015), arXiv:1406.5127 [hep-lat].

[25] M. Bander, Phys. Rept. 75, 205 (1981).

[26] J. Greensite, Prog. Part. Nucl. Phys. 51, 1 (2003), hep-lat/0301023.

[27] G. 't Hooft, in High Energy Physics, EPS International Conference, Palermo, 1975, edited by A. Zichichi (1975).

[28] S. Mandelstam, Phys. Rept. 23, 245 (1976).

[29] G. Ripka, Lect. Notes Phys. 639, 1 (2004).

[30] K.-I. Kondo, S. Kato, A. Shibata, and T. Shinohara, Phys. Rept. 579, 1 (2015), arXiv:1409.1599 [hep-th].

[31] H. Shiba and T. Suzuki, Phys. Lett. B351, 519 (1995), arXiv:hep-lat/9408004.

[32] N. Arasaki, S. Ejiri, S.-i. Kitahara, Y. Matsubara, and T. Suzuki, Phys. Lett. B395, 275 (1997), arXiv:hep-lat/9608129.

[33] P. Cea and L. Cosmai, Phys. Rev. D62, 094510 (2000), arXiv:hep-lat/0006007.

[34] P. Cea and L. Cosmai, JHEP 11, 064 (2001).

[35] A. Di Giacomo, B. Lucini, L. Montesi, and G. Paffuti, Phys. Rev. D61, 034503 (2000), arXiv:hep-lat/9906024. 
[36] A. Di Giacomo, B. Lucini, L. Montesi, and G. Paffuti, Phys. Rev. D61, 034504 (2000), arXiv:hep-lat/9906025.

[37] J. M. Carmona, M. D’Elia, A. Di Giacomo, B. Lucini, and G. Paffuti, Phys. Rev. D64, 114507 (2001), arXiv:hep-lat/0103005.

[38] P. Cea, L. Cosmai, and M. D'Elia, JHEP 02, 018 (2004), arXiv:hep-lat/0401020.

[39] A. D'Alessandro, M. D'Elia, and E. V. Shuryak, Phys.Rev. D81, 094501 (2010), arXiv:1002.4161 [hep-lat].

[40] S. Kato, K.-I. Kondo, and A. Shibata, Phys. Rev. D91, 034506 (2015), arXiv:1407.2808 [hep-lat].

[41] G. 't Hooft, (2004), hep-th/0408183.

[42] P. Cea, L. Cosmai, F. Cuteri, and A. Papa, JHEP 06, 033 (2016), arXiv:1511.01783 [hep-lat].

[43] M. Caselle, M. Panero, and D. Vadacchino, JHEP 02, 180 (2016), arXiv:1601.07455 [hep-lat].

[44] M. Baker, Phys. Rev. D93, 054012 (2016), arXiv:1512.02705 [hep-ph].

[45] M. Luscher, Nucl. Phys. B180, 317 (1981).

[46] M. Luscher, K. Symanzik, and P. Weisz, Nucl. Phys. B173, 365 (1980).

[47] M. Luscher, G. Munster, and P. Weisz, Nucl. Phys. B180, 1 (1981).

[48] M. Caselle, M. Pepe, and A. Rago, JHEP 10, 005 (2004), arXiv:hep-lat/0406008 [hep-lat].

[49] M. Caselle, M. Hasenbusch, and M. Panero, JHEP 03, 026 (2005), arXiv:hep-lat/0501027 [hep-lat].

[50] M. Caselle, P. Grinza, and N. Magnoli, J. Stat. Mech. 0611, P11003 (2006), arXiv:hepth/0607014 [hep-th].

[51] F. Gliozzi, M. Pepe, and U. J. Wiese, JHEP 01, 057 (2011), arXiv:1010.1373 [hep-lat].

[52] F. Gliozzi, M. Pepe, and U. J. Wiese, Phys. Rev. Lett. 104, 232001 (2010), arXiv:1002.4888 [hep-lat].

[53] M. Caselle, A. Feo, M. Panero, and R. Pellegrini, JHEP 04, 020 (2011), arXiv:1102.0723 [hep-lat].

[54] M. Caselle and P. Grinza, JHEP 1211, 174 (2012), arXiv:1207.6523 [hep-th].

[55] A. S. Bakry, D. B. Leinweber, and A. G. Williams, Proceedings, 29th International Symposium on Lattice field theory (Lattice 2011), PoS LATTICE2011, 256 (2011).

[56] A. S. Bakry, X. Chen, and P.-M. Zhang, Phys. Rev. D91, 114506 (2015), arXiv:1412.3568 [hep-lat]. 
[57] A. S. Bakry, D. B. Leinweber, and A. G. Williams, Proceedings, 30th International Symposium on Lattice Field Theory (Lattice 2012), PoS LATTICE2012, 271 (2012).

[58] A. S. Bakry, D. B. Leinweber, and A. G. Williams, Phys. Rev. D91, 094512 (2015), arXiv:1107.0150 [hep-lat].

[59] A. S. Bakry, D. B. Leinweber, and A. G. Williams, T(r)opical QCD II. Proceedings, 3rd Workshop, Cairns, Australia, September 26-October 1, 2010, AIP Conf. Proc. 1354, 178 (2011).

[60] A. S. Bakry, D. B. Leinweber, P. J. Moran, A. Sternbeck, and A. G. Williams, Phys. Rev. D82, 094503 (2010), arXiv:1004.0782 [hep-lat].

[61] D. S. Kuzmenko and Y. A. Simonov, Phys. Lett. B494, 81 (2000), arXiv:hep-ph/0006192.

[62] A. Di Giacomo, H. G. Dosch, V. I. Shevchenko, and Y. A. Simonov, Phys. Rept. 372, 319 (2002), arXiv:hep-ph/0007223.

[63] A. Di Giacomo and E. Meggiolaro, Phys. Lett. B537, 173 (2002), arXiv:hep-lat/0203012 [hep-lat].

[64] J. R. Clem, Journal of Low Temperature Physics 18, 427 (1975), 10.1007/BF00116134.

[65] http://physics.utah.edu/ detar/milc.html.

[66] R. G. Edwards, U. M. Heller, and T. R. Klassen, Nucl. Phys. B517, 377 (1998), heplat/9711003.

[67] E. Eichten, K. Gottfried, T. Kinoshita, K. D. Lane, and T. M. Yan, Phys. Rev. D 21, 203 (1980).

[68] A. Bazavov et al. (MILC), Phys. Rev. D82, 074501 (2010), arXiv:1004.0342 [hep-lat].

[69] A. Bazavov et al., Phys. Rev. D85, 054503 (2012), arXiv:1111.1710 [hep-lat].

[70] A. Hasenfratz and F. Knechtli, Phys. Rev. D64, 034504 (2001), arXiv:hep-lat/0103029 [heplat].

[71] M. Falcioni, M. Paciello, G. Parisi, and B. Taglienti, Nuclear Physics B 251, 624 (1985).

[72] P. Bicudo, N. Cardoso, and M. Cardoso, (2017), arXiv:1702.03454 [hep-lat].

[73] G. S. Bali, H. Neff, T. Duessel, T. Lippert, and K. Schilling (SESAM), Phys. Rev. D71, 114513 (2005), arXiv:hep-lat/0505012 [hep-lat].

[74] S. Kratochvila and P. de Forcrand, Lattice field theory. Proceedings: 20th International Symposium, Lattice 2002, Cambridge, USA, Jun 24-29, 2002, Nucl. Phys. Proc. Suppl. 119, 670 (2003), [,670(2002)], arXiv:hep-lat/0209094 [hep-lat]. 
[75] V. Koch, J. Bulava, B. Hörz, F. Knechtli, G. Moir, C. Morningstar, and M. Peardon, Proceedings, 33rd International Symposium on Lattice Field Theory (Lattice 2015): Kobe, Japan, July 14-18, 2015, PoS LATTICE2015, 100 (2016), arXiv:1511.04029 [hep-lat]. 